\title{
A Maximum Likelihood Approach for SSS Detection in LTE Systems
}

\author{
Michele Morelli, Senior Member, IEEE, and Marco Moretti, Member, IEEE
}

\begin{abstract}
Before establishing a communication link with the serving base station (eNodeB), a user equipment (UE) operating in a long-term evolution (LTE) multi-cellular network must acquire some specific information, including the sector identity and cell group identity. For this purpose, two training sequences called primary synchronization signal (PSS) and secondary synchronization signal (SSS) are periodically transmitted in the downlink to convey such information. In this work, we present a novel maximum likelihood (ML) approach for SSS detection assuming that the PSS has been successfully identified at an earlier stage. As we shall see, the resulting scheme turns out to be too complex for practical implementation as it requires perfect knowledge of the channel covariance matrix. Therefore, we look for simpler solutions and propose two reduced-search methods that operate in a mismatched mode. The first scheme exploits channel state information emerging from both the primary and secondary synchronization signals, while the second scheme operates using only the secondary synchronization signal.

Numerical analysis indicates that the proposed methods outperform existing alternatives and can be successfully applied even in a severe propagation scenario. The price for such an advantage is a certain increase of the processing requirement.
\end{abstract}

Index Terms-Long-term evolution, secondary synchronization sequence, cell group identity.

\section{INTRODUCTION}

The long term evolution (LTE) is a mobile communication standard released by the third generation partnership project (3GPP) in order to increase the spectrum efficiency and data throughput of $3 \mathrm{G}$ systems [1]. Orthogonal frequency-division multiple-access (OFDMA) is adopted in the downlink as an air interface in order to improve resilience against multipath distortions, while single-carrier frequency-division multipleaccess (SC-FDMA) is used in the uplink to reduce the peakto-average power-ratio at the UE.

LTE supports multi-cell communications, with the cell identity being specified by a couple of integer numbers, called sector ID and cell group ID. Upon entering the network, the UE must activate the cell search procedure in order to acquire carrier frequency offset, frame boundary and cell identity information [2]. For this purpose, the eNodeB employs a specified set of subcarriers to periodically transmit two pilot sequences, known as the primary synchronization signal (PSS) and secondary synchronization signal (SSS). The former is a Zadoff-Chu sequence specifying the sector ID, while the latter is a concatenation of two scrambled maximal length sequences ( $m$-sequences) placed at even and odd subcarriers, whose cyclic shifts univocally determine the cell group ID.

Considering that a total of 168 different combinations of cyclic shifts are envisaged in LTE, reliable SSS detection is a

This work has been supported by the PRA 2016 research project 5GIOTTO funded by the University of Pisa. non-trivial task which has been extensively studied in the last few years. As a result, many solutions are currently available. Some of them rely on the observation of two consecutive SSS time slots in order to improve the detection capability [3]-[6]. This approach, however, results into a prolonged acquisition time since only one SSS is inserted in each LTE half-frame. Alternative schemes that exploit a single SSS time slot can be found in [7]-[10]. Although the best performance in terms of detection capability is achieved through a complete search over all possible 168 hypotheses, most existing algorithms adopt a reduced-complexity approach wherein the cyclic shifts of the two $m$-sequences are detected sequentially instead of jointly. For example, in [7] the unknown shifts are found by correlating the odd and even subcarriers of the received SSS with replicas of the descrambled $m$-sequences. An underlying assumption behind this method is that the channel is approximately flat over the SSS subcarriers and, therefore, some performance degradation is expected in the presence of severe multipath propagation and non-negligible timing errors, which appear as a linear phase shift of the channel frequency response (CFR) across the signal spectrum. To improve the resilience against channel selectivity and timing errors, a differential SSS detector is presented in [8]. Compared to [7], however, the differential approach can reduce the accuracy at low signal-to-noise ratios (SNRs) or in the presence of nearly-flat channels. A possible trade-off between coherent and differential detection is found in [8] by resorting to the partial correlation concept, where the frequency interval occupied by the SSS subcarriers is fragmented into adjacent subbands, which are correlated with the corresponding parts of the hypothesized $m$-sequence. An efficient approach to cope with channel selectivity is represented by coherent SSS detection. In such a case, an estimate of the CFR is obtained at the PSS position [11], [12] and used to compensate for channel distortions over the SSS time-slot [8], [9]. This solution is particularly suited for LTE systems operating in the frequencydivision-duplexing (FDD) mode, where the PSS and SSS slots are close in time. In contrast, in the time-division-duplexing (TDD) mode the synchronization sequences are separated by two OFDM symbols and may experience different channel distortions, thereby reducing the advantage of the coherent approach.

In this paper we study the problem of SSS detection in an LTE system operating in either the FDD or TDD mode. In doing so, we assume that frequency synchronization and half-frame timing alignment have been successfully completed at an earlier stage using the recovered PSS. In contrast to previous investigations, we follow a rigorous approach based on the maximum likelihood (ML) estimation principle where 
the channel covariance matrix is used to characterize the distortions introduced by the propagation medium. Unfortunately, the resulting ML algorithm is not suited for practical implementation as it requires perfect knowledge of the channel statistics, which should be estimated in some manner. For this reason, we adopt a simplified approach and propose two SSS detection methods that operate in a mismatched mode. One of them takes advantage of the channel state information retrieved from the PSS time slot, while the other one detects the cell group ID by only exploiting the received SSS. Conventional reduced-search techniques can be applied to further reduce the processing requirement.

The rest of the paper is organized as follows. Next Section illustrates the structure of the LTE synchronization sequences and summarizes the cell search procedure. Sect. III presents the signal model and formulates the estimation problem. The ML estimator of the cell group ID is derived in Sect. IV, where some heuristic adjustments are also suggested to facilitate its practical implementation. After reviewing conventional SSS detection methods in Sect. V, we discuss simulation results in Sect. VI and, finally, we draw some conclusions in Sect. VII.

Notation: Matrices and vectors are denoted by boldface letters, with $\mathbf{I}_{N}$ being the identity matrix of order $N$ and $\mathbf{A}=\operatorname{diag}\{a(n) ; n=1,2, \ldots, N\}$ indicating an $N \times N$ diagonal matrix with entries $a(n)$ along its main diagonal. We use $\mathrm{E}\{\cdot\},(\cdot)^{*},(\cdot)^{T}$ and $(\cdot)^{H}$ for expectation, complex conjugation, transposition and Hermitian transposition, respectively, while $\mathbf{B}^{-1}$ is the inverse of a matrix $\mathbf{B}$. The notation $\Re \mathrm{e}\{\cdot\}$ stands for the real part of a complex-valued quantity and $|\cdot|$ represents the corresponding modulus. We use $\lfloor c\rfloor$ to indicate the smallest integer larger than or equal to $c$, while $\delta(m)$ is the Kronecker delta function. Finally, we denote by $\tilde{\lambda}$ a trial value of an unknown parameter $\lambda$.

\section{LTE SYNCHRONIZATION SEQUENCES}

\section{A. LTE frame structure}

In LTE systems, data transmission is organized in $10 \mathrm{~ms}$ radio frames. Each frame is divided into ten $1 \mathrm{~ms}$ subframes, which are further partitioned into two slots of $0.5 \mathrm{~ms}$ duration. Cell identity information is conveyed by an integer number called cell-ID. The latter is uniquely specified as $N_{I D}^{\text {cell }}=$ $3 N_{I D}^{(1)}+N_{I D}^{(2)}$, where $N_{I D}^{(1)} \in\{0,1, \ldots, 167\}$ is the cell group ID and $N_{I D}^{(2)} \in\{0,1,2\}$ denotes the sector ID within a group. This means that there is a total of 504 cell identities, which are arranged into 168 different groups.

In order to facilitate the cell search task, two synchronization signals called PSS and SSS are broadcast by the eNodeB every $5 \mathrm{~ms}$ over a group of 72 adjacent subcarriers placed symmetrically around the DC. For the sake of brevity, in the sequel we denote such a group of subcarriers as the synchronization sub-band (SSB). In particular, the PSS is transmitted on the primary SSB (P-SSB) and specifies the sector ID $N_{I D}^{(2)}$, while the SSS is transmitted on the secondary SSB (S-SSB) and provides the cell group ID $N_{I D}^{(1)}$. The position of the synchronization sequences within the radio frame depends on the adopted duplexing mode. As shown in Fig. 1, in the type 1 FDD frame the PSS and SSS are located at the last and second last OFDM symbols of the first and 11 th slots. In the type 2 TDD frame structure, which is

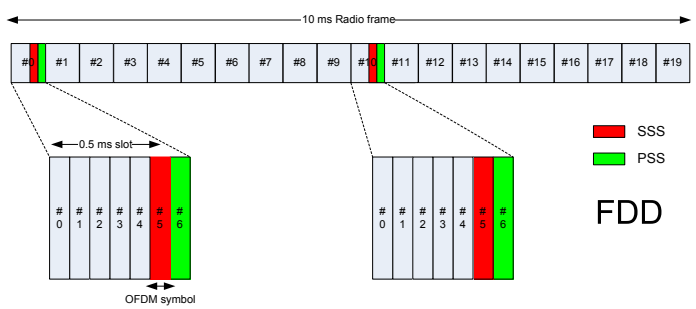

Fig. 1. Structure of the type1 FDD frame.

described in Fig. 2, the SSS is mapped to the last OFDM symbol of the second and 12th slots, while the PSS is three OFDM symbols apart from the SSS. It is worth noting that the SSS is transmitted every $5 \mathrm{~ms}$ in subframe 0 and subframe 5 in both the TDD and FDD modes.

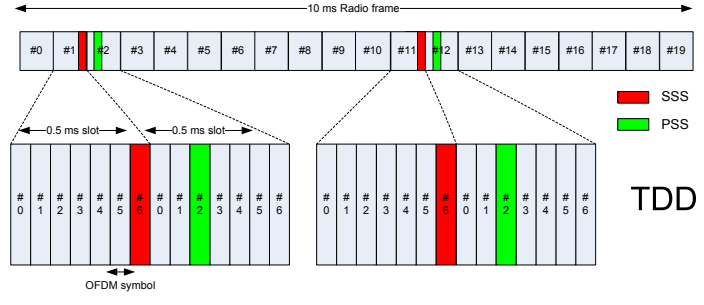

Fig. 2. Structure of the type2 TDD frame.

\section{B. PSS Structure}

The PSS is generated from a polyphase Zadoff-Chu (ZC) sequence defined as

$$
d_{P}(k)=\left\{\begin{array}{cc}
e^{-j \pi u k(k+1) / 63} & 0 \leq k \leq 30 \\
e^{-j \pi u(k+1)(k+2) / 63} & 31 \leq k \leq 61
\end{array}\right.
$$

where the root-index $u$ belongs to the set $\{25,29,34\}$ and univocally determines the sector ID $N_{I D}^{(2)}$ through a specified mapping rule. The choice of $\mathrm{ZC}$ sequences is driven by their excellent correlation properties and low sensitivity to Doppler frequency offset.

\section{SSS Structure}

The SSS is constructed from two scrambled and cyclicallyshifted binary sequences $\tilde{s}(k)$ and $\tilde{z}(k)$, with $k=0,1, \ldots, 30$. The arrangement differs between subframe 0 and subframe 5 according to

$$
\begin{gathered}
d_{S}(2 k)= \begin{cases}s_{m_{0}}(k) c_{0}(k) & \text { in subframe } 0 \\
s_{m_{1}}(k) c_{0}(k) & \text { in subframe } 5\end{cases} \\
d_{S}(2 k+1)= \begin{cases}s_{m_{1}}(k) c_{1}(k) z_{m_{0}}(k) & \text { in subframe } 0 \\
s_{m_{0}}(k) c_{1}(k) z_{m_{1}}(k) & \text { in subframe 5 }\end{cases}
\end{gathered}
$$

where $s_{m_{i}}(k)=\tilde{s}\left[\left(k+m_{i}\right) \bmod 31\right]$ and $z_{m_{i}}(k)=\tilde{z}[(k+$ $\left.\left.\left(m_{i}\right) \bmod 8\right) \bmod 31\right]$ for $i=0$ or 1 , while $c_{i}(k)=\tilde{c}[(k+$ $\left.N_{I D}^{(2)}+3 i\right)_{\bmod 31]}$ is the scrambling sequence. As specified in $\left[1\right.$, p. 95], the pair $\left(m_{0}, m_{1}\right)$ univocally determines $N_{I D}^{(1)}$. 
The sequences $\left\{d_{P}(k)\right\}$ and $\left\{d_{S}(k)\right\}$ are mapped onto the central 62 P-SSB and S-SSB subcarriers, respectively, with indices $\ell \in\{ \pm 1, \pm 2, \ldots, \pm 31\}$ specified by

$$
\ell=(k-31)+\lfloor k / 31\rfloor \quad k=0,1, \ldots, 61 .
$$

This means that a total of eleven SSB subcarriers (five placed at each subband boundary and one at DC) are left unmodulated.

\section{Summary of the cell search procedure}

In an LTE multi-cell communication system, cell search represents a fundamental procedure by which the UE can acquire information about the serving eNodeB. This task is typically broken into three hierarchical stages. In the first one, initial frequency and timing synchronization is achieved by exploiting the redundancy associated to the cyclic prefix $(\mathrm{CP})$ of the OFDM symbols [14]. This operation is accomplished in the time-domain and provides information about the start of each symbol. After frequency correction and $\mathrm{CP}$ removal, the received samples are converted in the frequency-domain using a discrete Fourier transform (DFT) device. The second stage is devoted to PSS detection, by which the ZC root index is retrieved together with the corresponding sector index. Upon successful completion of this task, the UE achieves $5 \mathrm{~ms}$ timing information and can determine the SSS position in the received downlink signal. The next step is the detection of the pair $\left(m_{0}, m_{1}\right)$ and the acquisition of frame synchronization by distinguishing between the following two hypotheses

$$
\begin{aligned}
& \mathcal{H}_{0}: \text { the detected SSS belongs to subframe } 0 \\
& \mathcal{H}_{1}: \text { the detected SSS belongs to subframe } 5
\end{aligned}
$$

Since there are 168 different pairs $\left(m_{0}, m_{1}\right)$ for each hypothesis $\mathcal{H}_{0}$ or $\mathcal{H}_{1}$, the search space has cardinality 336 , which makes SSS detection the most demanding task of the overall cell search procedure.

\section{Signal MODEL AND PROBLEM FORMULATION}

We assume that initial synchronization and PSS recovery have been successfully completed and concentrate on the SSS detection task. Since at this stage the position of the $\mathrm{P}-\mathrm{SSB}$ and S-SSB in the received frequency-domain signal is known, we extract the 62 PSS and SSS modulated subcarriers and arrange them into two vectors $\mathbf{U}_{P}$ and $\mathbf{U}_{S}$, respectively, with $\mathbf{U}_{P}=\left[U_{P}(0), U_{P}(1), \ldots, U_{P}(61)\right]^{T}$ and $\mathbf{U}_{S}=\left[U_{S}(0), U_{S}(1), \ldots, U_{S}(61)\right]^{T}$. Then, observing that the scrambling sequences $c_{i}(k)$ depend on $N_{I D}^{(2)}$, which is available after PSS detection, we compute a de-scrambled vector $\mathbf{Y}_{S}=\left[\begin{array}{ll}\mathbf{Y}_{S, \text { even }}^{T} & \mathbf{Y}_{S, \text { odd }}^{T}\end{array}\right]^{T}$, where $Y_{S, \text { even }}(n)=U_{S}(2 n) c_{0}(n)$ and $Y_{S, \text { odd }}(n)=U_{S}(2 n+1) c_{1}(n)$ for $n=0,1, \ldots, 30$. From (2) and (3), the entries of $\mathbf{Y}_{S}$ are found to be

$$
Y_{S}(n)=H_{S}\left(i_{n}\right) p(n ; a, b)+w_{S}(n) \quad n=0,1, \ldots, 61
$$

where $H_{S}\left(i_{n}\right)$ is the CFR at the $i_{n}$ th subcarrier of the S-SSB, $w_{S}(n)$ is white Gaussian noise and we have defined

$$
p(n ; a, b)=\left\{\begin{array}{cc}
s_{a}(n) & 0 \leq n \leq 30 \\
s_{b}(n) z_{a}(n) & 31 \leq n \leq 61
\end{array}\right.
$$

with

$$
(a, b)= \begin{cases}\left(m_{0}, m_{1}\right) & \text { subject to } \mathcal{H}_{0} \\ \left(m_{1}, m_{0}\right) & \text { subject to } \mathcal{H}_{1}\end{cases}
$$

Considering the mapping rule specified in (4), it follows that indices $n$ and $i_{n}$ are related by

$$
i_{n}=\left\{\begin{array}{lc}
2 n-31+\lfloor n / 16\rfloor & 0 \leq n \leq 30 \\
2 n-92+\lfloor n / 46\rfloor & 31 \leq n \leq 61 .
\end{array}\right.
$$

Inspection of (5) reveals that vector $\mathbf{Y}_{S}$ is affected by channel distortions, which can have a detrimental impact on the SSS detection capability. This problem is typically solved by resorting to either non-coherent differential detection or by coherent detection. The latter approach can be pursued by using channel estimates obtained form the recovered PSS. This is especially true in the FDD mode, where the S-SSB and P-SSB are arranged in adjacent OFDM symbols, so that channel variations are expected to be relatively small even in a high-mobility scenario. Channel estimates computed from the recovered PSS are collected into a 62-dimensional vector $\mathbf{Y}_{P}=\left[\begin{array}{ll}\mathbf{Y}_{P, \text { even }}^{T} & \mathbf{Y}_{P, \text { odd }}^{T}\end{array}\right]^{T}$, where

$$
\begin{aligned}
& Y_{P, \text { even }}(n)=U_{P}(2 n) d_{P}^{*}(2 n) \\
& Y_{P, \text { odd }}(n)=U_{P}(2 n+1) d_{P}^{*}(2 n+1)
\end{aligned} \quad \text { for } n=0,1, \ldots, 30 .
$$

The entries of $\mathbf{Y}_{P}$ are thus given by

$$
Y_{P}(n)=H_{P}\left(i_{n}\right)+w_{P}(n) \quad n=0,1, \ldots, 61
$$

where $H_{P}\left(i_{n}\right)$ is the CFR for the received P-SSB and $w_{P}(n)$ is still white Gaussian noise.

To proceed further, we denote by $\mathbf{h}_{P}=$ $\left[h_{P}(0), h_{P}(1), \ldots, h_{P}(L-1)\right]^{T}$ and $\mathbf{h}_{S}=\left[h_{S}(0), h_{S}(1)\right.$, $\left.\ldots, h_{S}(L-1)\right]^{T}$ the discrete-time channel impulse response (CIR) of order $L$ during the PSS and SSS transmission, respectively. Then, we can write the CFR vectors $\mathbf{H}_{P}=\left[H_{P}\left(i_{0}\right), H_{P}\left(i_{1}\right), \ldots, H_{P}\left(i_{61}\right)\right]^{T}$ and $\mathbf{H}_{S}=\left[H_{S}\left(i_{0}\right), H_{S}\left(i_{1}\right), \ldots, H_{S}\left(i_{61}\right)\right]^{T}$ as

$$
\begin{aligned}
\mathbf{H}_{P} & =\mathbf{F} \mathbf{h}_{P} \\
\mathbf{H}_{S} & =\mathbf{F} \mathbf{h}_{S}
\end{aligned}
$$

where $\mathbf{F}$ is a $62 \times L$ matrix with elements

$$
[\mathbf{F}]_{n, \ell}=e^{-j 2 \pi \ell i_{n} / N} \quad 0 \leq n \leq 61, \quad 0 \leq \ell \leq L-1
$$

and $N$ is the DFT size. Then, from (5) and (10) it turns out that $\mathbf{Y}_{P}$ and $\mathbf{Y}_{S}$ can be put in matrix form as

$$
\begin{gathered}
\mathbf{Y}_{P}=\mathbf{F} \mathbf{h}_{P}+\mathbf{w}_{P} \\
\mathbf{Y}_{S}=\mathbf{P}(a, b) \mathbf{F} \mathbf{h}_{S}+\mathbf{w}_{S}
\end{gathered}
$$

where $\mathbf{w}_{P}=\left[w_{P}(0), w_{P}(1), \ldots, w_{P}(61)\right]^{T}$ and $\mathbf{w}_{S}=$ $\left[w_{S}(0), w_{S}(1), \ldots, w_{S}(61)\right]^{T}$ are statistically independent zero-mean Gaussian vectors with covariance matrix $\sigma^{2} \mathbf{I}_{62}$, while

$$
\mathbf{P}(a, b)=\left[\begin{array}{cc}
\mathbf{S}(a) & \mathbf{0} \\
\mathbf{0} & \mathbf{S}(b) \mathbf{Z}(a)
\end{array}\right]
$$

with $\mathbf{S}(m)=\operatorname{diag}\left\{s_{m}(0), s_{m}(1), \ldots, s_{m}(30)\right\}(m=a, b)$ and $\mathbf{Z}(a)=\operatorname{diag}\left\{z_{a}(0), z_{a}(1), \ldots, z_{a}(30)\right\}$. Our goal is to exploit the observation vector $\mathbf{Y}=\left[\mathbf{Y}_{P}^{T} \mathbf{Y}_{S}^{T}\right]^{T}$ to get the ML estimate of the unknown parameters $(a, b)$. In doing so, we 
assume that the CIR vectors $\mathbf{h}_{P}$ and $\mathbf{h}_{S}$ in (13) are Gaussian distributed with zero-mean (Rayleigh fading), and adopt the typical wide-sense stationary uncorrelated scattering (WSSUS) model [15] such that

$$
\begin{aligned}
& \mathrm{E}\left\{h_{P}(\ell) h_{P}^{*}(\ell-m)\right\}=\mathrm{E}\left\{h_{S}(\ell) h_{S}^{*}(\ell-m)\right\}=\sigma_{\ell}^{2} \delta(m) \\
& \mathrm{E}\left\{h_{P}(\ell) h_{S}^{*}(\ell-m)\right\}=r_{t}\left(\tau_{1}-\tau_{0}\right) \sigma_{\ell}^{2} \delta(m) .
\end{aligned}
$$

In the above equations, $\sigma_{\ell}^{2}$ denotes the power of the $\ell$ th channel tap, $r_{t}(\tau)$ is the normalized time correlation function with $r_{t}(0)=1$ and, finally, $\tau_{1}-\tau_{0}$ is the time interval between the SSS and PSS reception. Inspection of (15) indicates that $\mathbf{h}_{P}$ and $\mathbf{h}_{S}$ have the same covariance matrix $\mathbf{C}_{\mathbf{h}}=\mathrm{E}\left\{\mathbf{h}_{P} \mathbf{h}_{P}^{H}\right\}=\mathrm{E}\left\{\mathbf{h}_{S} \mathbf{h}_{S}^{H}\right\}=\operatorname{diag}\left\{\sigma_{\ell}^{2} ; 0 \leq \ell \leq L-1\right\}$. Furthermore, we have $\mathbf{C}_{\mathbf{h}_{P} \mathbf{h}_{S}}=\mathrm{E}\left\{\mathbf{h}_{P} \mathbf{h}_{S}^{H}\right\}=\alpha \mathbf{C}_{\mathbf{h}}$, where $\alpha=r_{t}\left(\tau_{1}-\tau_{0}\right)$ is a decorrelating factor dependent on the Doppler bandwidth and duplexing mode. Denoting by $T_{B}$ the duration of the OFDM symbol (including the $\mathrm{CP}$ ) and assuming the Jakes' isotropic scattering model, from Figs. 1-2 it follows that

$$
\alpha= \begin{cases}J_{0}\left(2 \pi f_{D} T_{B}\right) & \text { for the FDD mode } \\ J_{0}\left(6 \pi f_{D} T_{B}\right) & \text { for the TDD mode }\end{cases}
$$

where $f_{D}$ is the maximum Doppler frequency and $J_{0}(x)$ the zero-th order Bessel function of the first kind.

\section{ESTIMATION OF THE UNKNOWN PARAMETERS}

\section{A. Derivation of the ML estimator}

The observation vector $\mathbf{Y}$ is expressed by

$$
\mathbf{Y}=\left[\begin{array}{c}
\mathbf{F h}_{P} \\
\mathbf{P}(a, b) \mathbf{F} \mathbf{h}_{S}
\end{array}\right]+\mathbf{w}
$$

where $\mathbf{w}=\left[\mathbf{w}_{P}^{T} \mathbf{w}_{S}^{T}\right]^{T}$. Assuming that the thermal noise $\mathbf{w}$ is statistically independent of the CIR vectors, it follows that $\mathbf{Y}$ is Gaussian distributed with zero-mean and covariance matrix $\mathbf{C}_{\mathbf{Y}}(a, b)=\mathbf{C}_{P}(a, b)+\sigma^{2} \mathbf{I}_{124}$, where $\mathbf{C}_{P}(a, b)$ is

$\mathbf{C}_{P}(a, b)=\left[\begin{array}{cc}\mathbf{F C}_{\mathbf{h}} \mathbf{F}^{H} & \alpha \mathbf{F} \mathbf{C}_{\mathbf{h}} \mathbf{F}^{H} \mathbf{P}^{T}(a, b) \\ \alpha \mathbf{P}(a, b) \mathbf{F} \mathbf{C}_{\mathbf{h}} \mathbf{F}^{H} & \mathbf{P}(a, b) \mathbf{F} \mathbf{C}_{\mathbf{h}} \mathbf{F}^{H} \mathbf{P}^{T}(a, b)\end{array}\right]$

so that the log-likelihood function (LLF) for the estimation of $(a, b)$ takes the form

$$
\Omega(\tilde{a}, \tilde{b})=-\ln \operatorname{det} \mathbf{C}_{\mathbf{Y}}(\tilde{a}, \tilde{b})-\mathbf{Y}^{H} \mathbf{C}_{\mathbf{Y}}^{-1}(\tilde{a}, \tilde{b}) \mathbf{Y} .
$$

To proceed further, we consider the eigenvalue decomposition of matrix $\mathbf{F} \mathbf{C}_{\mathbf{h}} \mathbf{F}^{H}$, i.e.,

$$
\mathbf{F C}_{\mathbf{h}} \mathbf{F}^{H}=\mathbf{U} \boldsymbol{\Lambda} \mathbf{U}^{H}
$$

where $\boldsymbol{\Lambda}=\operatorname{diag}\left\{\lambda_{1}, \lambda_{2}, \ldots, \lambda_{62}\right\}$ is a diagonal matrix containing the eigenvalues of $\mathbf{F C}_{\mathbf{h}} \mathbf{F}^{H}$ sorted in descending order, while the columns of $\mathbf{U}=\left[\begin{array}{llll}\mathbf{u}_{1} & \mathbf{u}_{2} & \cdots & \mathbf{u}_{62}\end{array}\right]$ are the corresponding eigenvectors. Substituting (20) into (18) and recalling that $\mathbf{U} \mathbf{U}^{H}=\mathbf{I}_{62}$, yields

$$
\mathbf{C}_{\mathbf{Y}}(\tilde{a}, \tilde{b})=\mathbf{Q}(\tilde{a}, \tilde{b})\left[\begin{array}{cc}
\boldsymbol{\Lambda} & \alpha \boldsymbol{\Lambda} \\
\alpha \boldsymbol{\Lambda} & \boldsymbol{\Lambda}
\end{array}\right] \mathbf{Q}^{H}(\tilde{a}, \tilde{b})+\sigma^{2} \mathbf{I}_{62}
$$

with

$$
\mathbf{Q}(\tilde{a}, \tilde{b})=\left[\begin{array}{cc}
\mathbf{U} & \mathbf{0} \\
\mathbf{0} & \mathbf{P}(\tilde{a}, \tilde{b}) \mathbf{U}
\end{array}\right] .
$$

Then, from the matrix determinant and matrix inversion lemmas applied to (21), it is found that

$$
\begin{gathered}
\ln \operatorname{det} \mathbf{C}_{\mathbf{Y}}(\tilde{a}, \tilde{b})=\sum_{k=1}^{62} \ln \left[\left(\lambda_{k}+\sigma^{2}\right)^{2}-\alpha^{2} \lambda_{k}^{2}\right] \\
\mathbf{C}_{\mathbf{Y}}^{-1}(\tilde{a}, \tilde{b})=\frac{1}{\sigma^{2}} \mathbf{I}_{124}-\frac{1}{\sigma^{2}} \mathbf{Q}(\tilde{a}, \tilde{b})\left[\begin{array}{cc}
\boldsymbol{\Gamma}_{0} & \boldsymbol{\Gamma}_{1} \\
\boldsymbol{\Gamma}_{1} & \boldsymbol{\Gamma}_{0}
\end{array}\right] \mathbf{Q}^{H}(\tilde{a}, \tilde{b})
\end{gathered}
$$

where $\boldsymbol{\Gamma}_{i}=\operatorname{diag}\left\{\gamma_{i}(1), \gamma_{i}(2), \ldots, \gamma_{i}(62)\right\}$ for $i=0,1$ and

$$
\begin{aligned}
& \gamma_{0}(k)=\frac{\lambda_{k}\left(\lambda_{k}-\alpha^{2} \lambda_{k}+\sigma^{2}\right)}{\left(\lambda_{k}+\sigma^{2}\right)^{2}-\alpha^{2} \lambda_{k}^{2}} \\
& \gamma_{1}(k)=\frac{\alpha \lambda_{k} \sigma^{2}}{\left(\lambda_{k}+\sigma^{2}\right)^{2}-\alpha^{2} \lambda_{k}^{2}} .
\end{aligned}
$$

Hence, letting $\mathbf{v}_{P}=\mathbf{U}^{H} \mathbf{Y}_{P}$ and $\mathbf{v}_{S}(\tilde{a}, \tilde{b})=\mathbf{U}^{H} \mathbf{P}(\tilde{a}, \tilde{b}) \mathbf{Y}_{S}$, after skipping irrelevant terms independent of $(\tilde{a}, \tilde{b})$, the LLF in (19) takes the form

$$
\Phi(\tilde{a}, \tilde{b})=\mathbf{v}_{S}^{H}(\tilde{a}, \tilde{b}) \boldsymbol{\Gamma}_{0} \mathbf{v}_{S}(\tilde{a}, \tilde{b})+2 \Re \operatorname{e}\left\{\mathbf{v}_{P}^{H} \boldsymbol{\Gamma}_{1} \mathbf{v}_{S}(\tilde{a}, \tilde{b})\right\}
$$

or, equivalently,

$$
\begin{aligned}
\Phi(\tilde{a}, \tilde{b})= & \sum_{k=1}^{62}\left[\gamma_{0}(k)\left|v_{S}(\tilde{a}, \tilde{b} ; k)\right|^{2}\right. \\
& \left.+2 \gamma_{1}(k) \Re \operatorname{e}\left\{v_{P}^{*}(k) v_{S}(\tilde{a}, \tilde{b} ; k)\right\}\right]
\end{aligned}
$$

where $v_{P}(k)$ and $v_{S}(\tilde{a}, \tilde{b} ; k)$ denote the $k$ th entry of $\mathbf{v}_{P}$ and $\mathbf{v}_{S}(\tilde{a}, \tilde{b})$, respectively. The ML estimate of $(a, b)$ is eventually obtained by locating the maximum of $\Phi(\tilde{a}, \tilde{b})$ over the 336 hypothesized values $(\tilde{a}, \tilde{b})$, yielding

$$
(\hat{a}, \hat{b})_{M L}=\arg \max _{(\tilde{a}, \tilde{b})}\{\Phi(\tilde{a}, \tilde{b})\} .
$$

Recalling that $m_{1}>m_{0}$, an estimate of $\left(m_{0}, m_{1}\right)$ is obtained from $(\hat{a}, \hat{b})$ as $\hat{m}_{0}=\min \{\hat{a}, \hat{b}\}$ and $\hat{m}_{1}=\max \{\hat{a}, \hat{b}\}$. Furthermore, from (7) it follows that a decision in favour of $\mathcal{H}_{0}$ is taken if $\hat{a}<\hat{b}$, while $\mathcal{H}_{1}$ is chosen if $\hat{a}>\hat{b}$.

\section{B. Practical adjustments}

Evaluating the ML metric $\Phi(\tilde{a}, \tilde{b})$ as indicated in (28) requires knowledge of the channel statistics and thermal noise. Since estimating these quantities would entail a remarkable increase of the computational load, we suggest an alternative approach based on the following practical adjustments.

1) The eigenvector matrix $\mathbf{U}$ employed for the evaluation of $\mathbf{v}_{P}$ and $\mathbf{v}_{S}(\tilde{a}, \tilde{b})$ depends on the channel covariance matrix $\mathbf{C}_{\mathbf{h}}$, which is generally unknown at the receiver. This problem can be circumvented by allowing the system to operate in a mismatched mode, wherein $\mathbf{C}_{\mathbf{h}}$ is replaced by some fixed matrix $\overline{\mathbf{C}}_{\mathbf{h}}$. A similar approach was adopted in [14] in the context of linear minimum mean square error (LMMSE) channel estimation for OFDM systems. In that case, a robust scheme providing good performance over a wide range of channel statistics was found by designing the estimator for a uniform power delay profile. This amounts to putting $\overline{\mathbf{C}}_{\mathbf{h}}=(1 / L) \mathbf{I}_{L}$, so that $\mathbf{F C}_{\mathbf{h}} \mathbf{F}^{H}$ becomes a known 
matrix $\mathbf{F} \overline{\mathbf{C}}_{\mathbf{h}} \mathbf{F}^{H}=(1 / L) \mathbf{F} \mathbf{F}^{H}$ with eigenvalue decomposition $\overline{\mathbf{U}} \overline{\mathbf{\Lambda}} \overline{\mathbf{U}}^{H}$. The mismatched eigenvector matrix $\overline{\mathbf{U}}$ is then employed to evaluate $\overline{\mathbf{v}}_{P}=\overline{\mathbf{U}}^{H} \mathbf{Y}_{P}$ and $\overline{\mathbf{v}}_{S}(\tilde{a}, \tilde{b})=\overline{\mathbf{U}}^{H} \mathbf{P}(\tilde{a}, \tilde{b}) \mathbf{Y}_{S}$, which are used in (27) in place of $\mathbf{v}_{P}$ and $\mathbf{v}_{S}(\tilde{a}, \tilde{b})$. Furthermore, the mismatched eigenvalues $\bar{\lambda}_{k}$ are used in (25) and (26) to get $\gamma_{0}(k)$ and $\gamma_{1}(k)$.

2) Inspection of (25) and (26) reveals that, after replacing $\lambda_{k}$ with the mismatched value $\bar{\lambda}_{k}$, the quantities $\alpha$ and $\sigma^{2}$ are still needed to complete the computation of $\gamma_{0}(k)$ and $\gamma_{1}(k)$. As shown in (16), parameter $\alpha$ depends on the maximum Doppler frequency $f_{D}$, which is related to the UE speed $v$ and to the carrier frequency $f_{0}$ by

$$
f_{D}=\frac{v f_{0}}{c}
$$

with $c$ being the speed of light. One possible approach is to design $\alpha$ for the maximum expected UE speed, say $v_{\max }$. This amounts to replacing $\alpha$ with

$$
\bar{\alpha}= \begin{cases}J_{0}\left(2 \pi \bar{f}_{D} T_{B}\right) & \text { FDD-mode } \\ J_{0}\left(6 \pi \bar{f}_{D} T_{B}\right) & \text { TDD-mode }\end{cases}
$$

where $\bar{f}_{D}=v_{\max } f_{0} / c$. As for the noise power $\sigma^{2}$, it can be estimated using the five unmodulated subcarriers placed at both edges of the P-SSB and S-SSB, yielding

$$
\hat{\sigma}^{2}=\frac{1}{20} \sum_{n \in \mathcal{J}}\left[\left|X_{P}(n)\right|^{2}+\left|X_{S}(n)\right|^{2}\right]
$$

where $\mathcal{J}=\{ \pm 32, \pm 33, \pm 34, \pm 35, \pm 36\}$ collects the indices of the SSB unmodulated subcarriers and $\left\{X_{P}(n), X_{S}(n)\right\}$ denotes the $n$th subcarrier of the PSSB and S-SSB, respectively. An alternative solution which dispenses with the estimation of $\sigma^{2}$ is obtained by designing $\gamma_{0}(k)$ and $\gamma_{1}(k)$ for some fixed nominal value $\sigma_{\text {nom }}^{2}$, corresponding to a specified signal-to-noise ratio (SNR). Putting together the above considerations, we can replace the coefficients $\gamma_{0}(k)$ and $\gamma_{1}(k)$ with some quantities $\bar{\gamma}_{0}(k)$ and $\bar{\gamma}_{1}(k)$, which are computed from (25) and (26) after substituting $\left\{\lambda_{k}, \alpha, \sigma^{2}\right\}$ with $\left\{\bar{\lambda}_{k}, \bar{\alpha}, \bar{\sigma}^{2}\right\}$, where $\bar{\sigma}^{2}$ is either $\hat{\sigma}^{2}$ or $\sigma_{\text {nom }}^{2}$ depending on the adopted strategy.

3) As shown later, the magnitude of the mismatched eigenvalues $\bar{\lambda}_{k}$ drops rapidly as $k$ increases. This means that most of the channel energy is conveyed by the first $K$ eigenvalues $\left\{\bar{\lambda}_{1}, \bar{\lambda}_{2}, \ldots, \bar{\lambda}_{K}\right\}$, where $K$ is a design parameter that depends on the channel order $L$. For example, for $L=1$ matrix $\mathbf{F} \overline{\mathbf{C}}_{\mathbf{h}} \mathbf{F}^{H}$ has all unit entries and its eigenvalues are $\bar{\lambda}_{1}=1$ and $\bar{\lambda}_{k}=0$ for $k \geq 2$, which corresponds to having $K=1$. Since the coefficients $\bar{\gamma}_{0}(k)$ and $\bar{\gamma}_{1}(k)$ are proportional to $\bar{\lambda}_{k}$, we can achieve some computational saving by letting $\bar{\gamma}_{0}(k)=\bar{\gamma}_{1}(k)=0$ for $k>K$. This yields the reducedrank and mismatched metric

$$
\begin{aligned}
\Psi(\tilde{a}, \tilde{b})= & \sum_{k=1}^{K}\left[\bar{\gamma}_{0}(k)\left|\bar{v}_{S}(\tilde{a}, \tilde{b} ; k)\right|^{2}\right. \\
& \left.+2 \bar{\gamma}_{1}(k) \Re \operatorname{e}\left\{\bar{v}_{P}^{*}(k) \bar{v}_{S}(\tilde{a}, \tilde{b} ; k)\right\}\right]
\end{aligned}
$$

which exploits only the first $K$ entries of vectors $\overline{\mathbf{v}}_{P}$ and $\overline{\mathbf{v}}_{S}(\tilde{a}, \tilde{b})$. Since $\overline{\mathbf{v}}_{P}$ is obtained from the channel estimates $\mathbf{Y}_{P}$ corresponding to the P-SSB, in the sequel the estimator based on the metric $\Psi(\tilde{a}, \tilde{b})$ is referred to as the coherent mismatched (CM) detector, i.e.,

$$
(\hat{a}, \hat{b})_{C M}=\arg \max _{(\tilde{a}, \tilde{b})}\{\Psi(\tilde{a}, \tilde{b})\} .
$$

4) From (25) and (26) we see that when $\bar{\alpha}=0$ the coefficients $\bar{\gamma}_{0}(k)$ and $\bar{\gamma}_{1}(k)$ takes the form

$$
\bar{\gamma}_{0}(k)=\frac{\bar{\lambda}_{k}}{\bar{\lambda}_{k}+\bar{\sigma}^{2}}, \quad \bar{\gamma}_{1}(k)=0
$$

and the metric $\Psi(\tilde{a}, \tilde{b})$ in (33) becomes

$$
\left.\Psi(\tilde{a}, \tilde{b})\right|_{\bar{\alpha}=0}=\sum_{k=1}^{K} \frac{\bar{\lambda}_{k}}{\bar{\lambda}_{k}+\bar{\sigma}^{2}}\left|\bar{v}_{S}(\tilde{a}, \tilde{b} ; k)\right|^{2} .
$$

In this case, channel estimates derived from the received P-SSB are not exploited to evaluate $\Psi(\tilde{a}, \tilde{b})$. The reason is that letting $\bar{\alpha}=0$ amounts to assuming that the CIR vectors $\mathbf{h}_{P}$ and $\mathbf{h}_{S}$ are uncorrelated and Gaussian distributed (hence, statistically independent), so that no useful information about $\mathbf{h}_{S}$ can be inferred from $\mathbf{Y}_{P}$. We denote the resulting estimator as the non-coherent mismatched (NCM) detector, i.e.,

$$
(\hat{a}, \hat{b})_{N C M}=\arg \max _{(\tilde{a}, \tilde{b})}\left\{\left.\Psi(\tilde{a}, \tilde{b})\right|_{\bar{\alpha}=0}\right\} .
$$

\section{Reduced-search (RS) estimation}

From the LTE specifications shown in [1, p. 95], it turns out that there are 168 different pairs $\left(m_{0}, m_{1}\right)$ for each hypothesis $\mathcal{H}_{0}$ or $\mathcal{H}_{1}$. This means that the search space spanned by $(\tilde{a}, \tilde{b})$ in the maximization problems (34) and (37) has cardinality 336. In order to reduce the processing load associated to such a large cardinality, we can adopt an approach similar to that suggested in [5], where the search space is reduced by decoupling the estimation of the two parameters $a$ and $b$. For this purpose, we let $\overline{\mathbf{U}}^{H}=\left[\overline{\mathbf{U}}_{\text {even }}^{H} \overline{\mathbf{U}}_{\text {odd }}^{H}\right]$, where $\overline{\mathbf{U}}_{\text {even }}^{H}$ and $\overline{\mathbf{U}}_{\text {odd }}^{H}$ are matrices of dimension $62 \times 31$ collecting the first 31 and the last 31 columns of $\overline{\mathbf{U}}^{H}$, respectively. Then, recalling that $\overline{\mathbf{v}}_{S}(\tilde{a}, \tilde{b})=\overline{\mathbf{U}}^{H} \mathbf{P}(\tilde{a}, \tilde{b}) \mathbf{Y}_{S}$, with $\mathbf{Y}_{S}=\left[\mathbf{Y}_{S, \text { even }}^{T}\right.$ $\left.\mathbf{Y}_{S, \text { odd }}^{T}\right]^{T}$ and $\mathbf{P}(\tilde{a}, \tilde{b})$ as given in (14), we have

$$
\overline{\mathbf{v}}_{S}(\tilde{a}, \tilde{b})=\overline{\mathbf{v}}_{S, \text { even }}(\tilde{a})+\overline{\mathbf{v}}_{S, \text { odd }}(\tilde{a}, \tilde{b})
$$

where

$$
\overline{\mathbf{v}}_{S, \text { even }}(\tilde{a})=\overline{\mathbf{U}}_{\text {even }}^{H} \mathbf{S}(\tilde{a}) \mathbf{Y}_{S, \text { even }}
$$

and

$$
\overline{\mathbf{v}}_{S, \text { odd }}(\tilde{a}, \tilde{b})=\overline{\mathbf{U}}_{\text {odd }}^{H} \mathbf{S}(\tilde{b}) \mathbf{Z}(\tilde{a}) \mathbf{Y}_{S, \text { odd }} .
$$

A RS estimator is found by replacing vector $\overline{\mathbf{v}}_{S}(\tilde{a}, \tilde{b})$ with $\overline{\mathbf{v}}_{S, \text { even }}(\tilde{a})$ in the CM metric (33). This yields the RS-CM estimator of $a$ in the form

$$
\begin{aligned}
\hat{a}_{R S-C M}=\arg \max _{\tilde{a}}\{ & \sum_{k=1}^{K}\left[\bar{\gamma}_{0}(k)\left|\bar{v}_{S, \text { even }}(\tilde{a}, k)\right|^{2}\right. \\
& \left.\left.+2 \bar{\gamma}_{1}(k) \Re \mathrm{e}\left\{\bar{v}_{P}^{*}(k) \bar{v}_{S, \text { even }}(\tilde{a} ; k)\right\}\right]\right\}
\end{aligned}
$$


where $\tilde{a} \in\{0,1, \ldots, 30\}$ and $\bar{v}_{S, \text { even }}(\tilde{a} ; k)$ is the $k$ th entry of $\overline{\mathbf{v}}_{S, \text { even }}(\tilde{a})$. Once $\hat{a}_{R S-C M}$ have been computed, it is used to get an estimate of $b$ as

$$
\hat{b}_{R S-C M}=\arg \max _{\tilde{b}}\left\{\Psi\left(\hat{a}_{R S-C M}, \tilde{b}\right)\right\}
$$

with $\Psi(\tilde{a}, \tilde{b})$ as given in (33). The search space for the maximization (42) depends on the value of $\hat{a}_{R S-C M}$ and can be written as $\tilde{b} \in J(\hat{a})$, where $\hat{a}=\hat{a}_{R S-C M}$ has been used to simplify the notation. Inspection of [1, p. 95] reveals that $J(\hat{a})$ is a subset of $\{\hat{a} \pm 1, \hat{a} \pm 2, \ldots, \hat{a} \pm 7\}$ for any value of $\hat{a}$. In particular, its cardinality $|J(\hat{a})|$ varies from a minimum of 6 (when $\hat{a}=30$ ) to a maximum of 13 (when $\hat{a}=7,8$ or 9 ), with an average value close to 11 . Hence, in the RS approach the number of test hypotheses $(\tilde{a}, \tilde{b})$ passes from 336 to an expected value of $N_{b}=42$, thereby leading to a substantial computational saving. As shown later by means of numerical analysis, this advantage comes at the cost of some performance degradation with respect to the complete-search algorithm.

The RS strategy can also be applied to the NCM algorithm. Bearing in mind the metric (36), the RS-NCM detector can be formulated as

$$
\begin{gathered}
\hat{a}_{R S-N C M}=\arg \max _{\tilde{a}}\left\{\sum_{k=1}^{K} \frac{\bar{\lambda}_{k}\left|\bar{v}_{S, \text { even }}(\tilde{a} ; k)\right|^{2}}{\bar{\lambda}_{k}+\bar{\sigma}^{2}}\right\} \\
\hat{b}_{R S-N C M}=\arg \max _{\tilde{b}}\left\{\sum_{k=1}^{K} \frac{\bar{\lambda}_{k}\left|\bar{v}_{S}\left(\hat{a}_{R S-N C M}, \tilde{b} ; k\right)\right|^{2}}{\bar{\lambda}_{k}+\bar{\sigma}^{2}}\right\} .
\end{gathered}
$$

\section{Complexity analysis}

The computational load of the complete-search detectors is dominated by the evaluation of the quantities $\left\{\bar{v}_{S}(\tilde{a}, \tilde{b} ; k) ; 1 \leq\right.$ $k \leq K\}$ for all 336 different pairs $(\tilde{a}, \tilde{b})$. An efficient way to compute $\bar{v}_{S}(\tilde{a}, \tilde{b} ; k)$ is based on the decomposition (38), which is reformulated as

$\bar{v}_{S}(\tilde{a}, \tilde{b} ; k)=\bar{v}_{S, \text { even }}(\tilde{a} ; k)+\bar{v}_{S, \text { odd }}(\tilde{a}, \tilde{b} ; k) \quad k=1,2, \ldots, K$.

From (39) we see that evaluating $\bar{v}_{S, \text { even }}(\tilde{a} ; k)$ for $k=$ $1,2, \ldots, K$ needs $31 \times K$ complex multiplications plus $30 \times K$ complex additions for each value of $\tilde{a}$, which corresponds to $246 \times K$ floating point operations (flops). In writing these figures we have borne in mind that a complex multiplication amounts to four real multiplications plus two real additions, while a complex additions is equivalent to two real additions. Furthermore, we have assumed that matrix $\overline{\mathbf{U}}$ is pre-computed and we have ignored the multiplication of $\mathbf{Y}_{S \text {, even }}$ by $\mathbf{S}(\tilde{a})$ as this operation only involves a sign inversion of some entries of $\mathbf{Y}_{S \text {,even }}$. Applying the same considerations to (40), it turns out that $246 \times K$ flops are required to compute the quantities $\left\{\bar{v}_{S, \text { odd }}(\tilde{a}, \tilde{b} ; k) ; 1 \leq k \leq K\right\}$ for each pair $(\tilde{a}, \tilde{b})$. Hence, recalling that $\tilde{a} \in\{0,1, \ldots, 30\}$ while $(\tilde{a}, \tilde{b})$ can take 336 different values, from (45) it follows that the complexity involved in the computation of $\left\{\bar{v}_{S}(\tilde{a}, \tilde{b} ; k) ; 1 \leq k \leq K\right\}$ for all admissible pairs $(\tilde{a}, \tilde{b})$ amounts to $91 \times K$ kflops. In order to evaluate the CM metric $\Psi(\tilde{a}, \tilde{b})$ in (33), it is also necessary to get the first $K$ entries of $\overline{\mathbf{v}}_{P}=\overline{\mathbf{U}}^{H} \mathbf{Y}_{P}$. However, since this operation is accomplished with $62 \times K$ complex multiplications plus $61 \times K$ complex additions (corresponding to $500 \times K$ flops), its impact on the overall complexity of the CM detector is quite marginal. Collecting the above results and bearing in mind that, once the quantities $\left\{\bar{v}_{S}(\tilde{a}, \tilde{b} ; k) ; 1 \leq k \leq K\right\}$ have been obtained, additional $3400 \times K$ flops and $1700 \times K$ flops are required to complete the computation of the metrics shown in (33) and (36), we conclude that SSS detection by means of $\mathrm{CM}$ and NCM approximately requires $95 \times K$ and $93 \times K$ kflops, respectively.

The computational load is much smaller if we adopt the RS approach. In such a case, computing the quantities $\left\{\bar{v}_{S, \text { even }}(\tilde{a}, k) ; 1 \leq k \leq K\right\}$ for $\tilde{a} \in\{\underset{\sim}{0}, 1, \ldots, 30\}$ needs $7.6 \times K$ kflops, while computing $\left\{\bar{v}_{S}(\hat{a}, \tilde{b} ; k) ; 1 \leq k \leq K\right\}$ for $\tilde{b} \in J(\hat{a})$ involves, on average, $2.7 \times K \mathrm{kflops}$. Once the quantities $\left\{\bar{v}_{S, \text { even }}(\tilde{a} ; k) ; 1 \leq k \leq K\right\}$ are available, $310 \times K$ flops are needed to evaluate the RS-CM metric in (41) for $\tilde{a} \in\{0,1, \ldots, 30\}$, while additional $110 \times K$ flops are required (on average) to complete the evaluation of $\Psi\left(\hat{a}_{R S-C M}, \tilde{b}\right)$ for $\tilde{b} \in J\left(\hat{a}_{R S-C M}\right)$. This leads to an overall complexity of $10.7 \times K$ kflops for the RS-CM. We also note that evaluation of the RS-NCM metric in (43) starting from $\left\{\bar{v}_{S, \text { even }}(\tilde{a} ; k) ; 1 \leq k \leq K\right\}$ requires $155 \times K$ flops, while computing the metric in (44) for $\tilde{b} \in J\left(\hat{a}_{R S-N C M}\right)$ starting from $\left\{\bar{v}_{S}\left(\hat{a}_{R S-N C M}, \tilde{b} ; k\right) ; 1 \leq k \leq K\right\}$ involves (on average) additional $75 \times K$ flops. It follows that approximately $10.5 \times K$ kflops are required for SSS detection by means of RS-NCM.

The overall complexity of all the considered schemes is summarized in Table I. As is seen, the RS methods entail a reduction of the overall complexity by approximately a factor of 9 with respect to their complete-search counterparts.

TABLE I

COMPLEXITY OF THE INVESTIGATED SCHEMES IN TERMS OF NUMBER OF FLOPS.

\begin{tabular}{|l|l||l|l|}
\hline $\begin{array}{l}\text { Algorithms } \\
\text { employing P-SSB } \\
\text { and S-SSB }\end{array}$ & $\begin{array}{l}\text { Required } \\
\text { number of } \\
\text { operations } \\
\text { (kflops) }\end{array}$ & $\begin{array}{l}\text { Algorithms } \\
\text { employing S-SSB } \\
\text { only }\end{array}$ & $\begin{array}{l}\text { Required } \\
\text { number of } \\
\text { operations } \\
\text { (kflops) }\end{array}$ \\
\hline \hline CM & $95 \times K$ & NCM & $93 \times K$ \\
\hline RS-CM & $10.7 \times K$ & RS-NCM & $10.5 \times K$ \\
\hline RS-CD & 10.5 & RS-MEJXD & 2.65 \\
\hline RS-MKBK & 5.2 & RS-DD & 10.1 \\
\hline
\end{tabular}

\section{REVIEW OF EXISTING SSS DETECTION SCHEMES}

It is interesting to compare the illustrated SSS detection algorithms with other existing solutions. Since the completesearch approach entails a remarkable computational load, most available schemes adopt the RS strategy. Among them, we briefly review the algorithm proposed by Manolakis, Estévez, Jungnickel, $\mathrm{Xu}$ and Drewes (RS-MEJXD) in [7] and the estimator proposed by Myung, Kang, Baek and Koo (RSMKBK) in [9]. We also discuss the differential and coherent detectors presented in [8], denoted by RS-DD and RS-CD, respectively. The complexity of the considered schemes is summarized in Table I. 


\section{A. The RS-MEJXD and $R S-D D$ solutions}

These schemes provide an estimate of $(a, b)$ without exploiting any channel state information from the received P-SSB. Parameter $a$ is firstly recovered by looking for the maximum of the metrics

$$
\begin{gathered}
\Gamma_{R S-M E J X D}(\tilde{a})=\left|\sum_{n=0}^{30} Y_{S, \text { even }}(n) s_{\tilde{a}}(n)\right|^{2} \\
\Gamma_{R S-D D}(\tilde{a})=\mid \sum_{n=0}^{29} Y_{S, \text { even }}(n) Y_{S, \text { even }}^{*}(n+1) \\
\times\left. s_{\tilde{a}}(n) s_{\tilde{a}}(n+1)\right|^{2}
\end{gathered}
$$

with respect to $\tilde{a} \in\{0,1, \ldots, 30\}$. Once the estimate of $a$ (say $\hat{a}$ ) is available, parameter $b$ is retrieved by maximizing the metrics

$$
\begin{gathered}
\Phi_{R S-M E J Z D}(\tilde{b})=\left|\sum_{n=0}^{30} Y_{S, \text { odd }}(n) s_{\tilde{b}}(n) z_{\hat{a}}(n)\right|^{2} \\
\Phi_{R S-D D}(\tilde{b})=\mid \sum_{n=0}^{29} Y_{S, \text { odd }}(n) Y_{S, \text { odd }}^{*}(n+1) \\
\times\left. s_{\tilde{b}}(n) s_{\tilde{b}}(n+1) z_{\hat{a}}(n) z_{\hat{a}}(n+1)\right|^{2}
\end{gathered}
$$

with respect to $\tilde{b} \in J(\hat{a})$. It is worth noting that the RS$\mathrm{NCM}$ metric shown in (43) reduces to $\Gamma_{R S-M E J Z D}(\tilde{a})$ in case of a flat channel of order $L=1$. In fact, in this case matrix $(1 / L) \mathbf{F} \mathbf{F}^{H}$ has only one non-zero eigenvalue with the associated eigenvector containing all unit entries. Therefore, in (43) we have $K=1$ with $\bar{v}_{S \text {,even }}(\tilde{a} ; 1)$ expressed by

$$
\bar{v}_{S, \text { even }}(\tilde{a} ; 1)=\sum_{n=0}^{30} Y_{S, \text { even }}(n) s_{\tilde{a}}(n) .
$$

\section{B. The RS-CD and RS-MKBK solutions}

In these schemes, coherent SSS detection is obtained by exploiting an estimate of the CFR obtained from the received PSS. The metrics used to retrieve parameter $a$ are expressed by

$$
\begin{aligned}
\Gamma_{R S-C D}(\tilde{a}) & =\left|\sum_{n=0}^{30} Y_{P, \text { even }}^{*}(n) Y_{S, \text { even }}(n) s_{\tilde{a}}(n)\right|^{2} \\
\Gamma_{R S-M K B K}(\tilde{a}) & =\Re \mathrm{e}\left\{\sum_{n=0}^{30} Y_{S, \text { even }}(n) s_{\tilde{a}}(n) / Y_{P, \text { even }}(n)\right\}_{(52)}
\end{aligned}
$$

while $b$ is found by looking for the maximum of the metrics

$$
\begin{gathered}
\Phi_{R S-C D}(\tilde{b})=\left|\sum_{n=0}^{30} Y_{P, \text { odd }}^{*}(n) Y_{S, \text { odd }}(n) s_{\tilde{b}}(n) z_{\hat{a}}(n)\right|^{2} \text {. 53) } \\
\Phi_{R S-M K B K}(\tilde{b})=\Re \mathrm{e}\left\{\sum_{n=0}^{30} Y_{S, \text { odd }}(n) s_{\tilde{b}}(n) z_{\hat{a}}(n) / Y_{P, \text { odd }}(n)\right\} .
\end{gathered}
$$

\section{Simulation RESUlts}

Computer simulations have been run to assess the performance of the presented SSS detection algorithms in an LTE communication system. The simulation parameters are chosen in agreement with the 3GPP specifications and are summarized as follows [1].

\section{A. Simulation model}

We consider a 20MHz LTE system operating in the 2.6 $\mathrm{GHz}$ frequency band. The DFT size is $N=2048$ and the sampling frequency is $f_{s}=30.72 \mathrm{MHz}$, corresponding to a $15 \mathrm{kHz}$ subcarrier spacing. The propagation scenarios are compliant with the Extended Typical Urban (ETU) and Extended Vehicular A (EVA) channel models. In both cases, the channel response is characterized by 9 multipath components with a maximum excess delay $\tau_{\max }$ of $5 \mu \mathrm{s}$ in the ETU scenario and $2.51 \mu \mathrm{s}$ for EVA. The path gains are generated by passing statistically independent and circularly symmetric white Gaussian processes through a fourth-order low-pass Butterworth filter. The 3-dB bandwidth of the filter is taken as a measure of the maximum Doppler frequency $f_{D}$, which is related to the UE speed $v$ and to the carrier frequency $f_{0}$ as indicated in (30). To demonstrate the capability of the proposed schemes in a high mobility scenario, we let $v=300$ $\mathrm{km} / \mathrm{h}$ in the ETU scenario, while $v=60 \mathrm{~km} / \mathrm{h}$ is adopted with the EVA. Pulse shaping is performed through a raisedcosine function with roll-off $\alpha=0.22$ and time-duration of 6 sampling periods, which corresponds to an overall CIR order of $L=\operatorname{int}\left\{f_{s} \tau_{\max }+6\right\}$. Although this parameter achieves a maximum of $L_{\max }=160$ in connection with the ETU scenario, the value $L=120$ is adopted for the design of the proposed SSS detection schemes. As explained in [11], this choice is motivated by the fact that a smaller value of $L$ can result into substantial computational saving by reducing the number $K$ of significant eigenvalues of matrix $\mathbf{F} \overline{\mathbf{C}}_{h} \mathbf{F}^{H}$. Since accurate timing information is difficult to achieve in a highly dispersive propagation environment [16], a residual timing error $\theta$ (normalized by the sampling period $T_{s}=1 / f_{s}$ ) is also included in our simulations. Unless otherwise specified, we let $\theta=40$.

Without any loss of generality, we consider the normal CP transmission mode, wherein 7 OFDM symbols are arranged in each slot. The first OFDM symbol in the slot has length $T_{B}^{(1)}=$ $71.86 \mu$ s (including the $\mathrm{CP}$ ), while the other 6 symbols have a shorter duration $T_{B}=71.36 \mu \mathrm{s}$. In order to make the system robust against channel variations, parameter $\bar{\alpha}$ is designed for a worst-case scenario wherein the maximum UE speed is set to $300 \mathrm{~km} / \mathrm{h}$, which leads to $\bar{f}_{D} T_{B}=v_{\max } f_{0} T_{B} / c \simeq 0.052$. Hence, from (31) it follows that $\bar{\alpha}=0.96$ in the FDD-mode, while $\bar{\alpha}=0.77$ in the TDD-mode. Parameter $\bar{\sigma}^{2}$ is fixed to a nominal value $\sigma_{\text {nom }}^{2}=0.1$. The accuracy of the SSS detection schemes is measured in terms of their failure probability, which is defined as $P_{f}=\operatorname{Pr}\left\{\left(\hat{m}_{0}, \hat{m}_{1}\right) \neq\left(m_{0}, m_{1}\right)\right\}$.

\section{B. Performance assessment}

An important design parameter for the proposed SSS detection schemes is the number $K$ of significant eigenvalues of 
the mismatched channel covariance matrix $(1 / L) \mathbf{F F}^{H}$. Fig. 3 illustrates the first fifteen eigenvalues $\bar{\lambda}_{k}(k=1,2, \ldots, 15)$ sorted in descending order with $L$ fixed to 120 . As is seen, only five of them are relevant, while all the others take very small values and can be neglected.

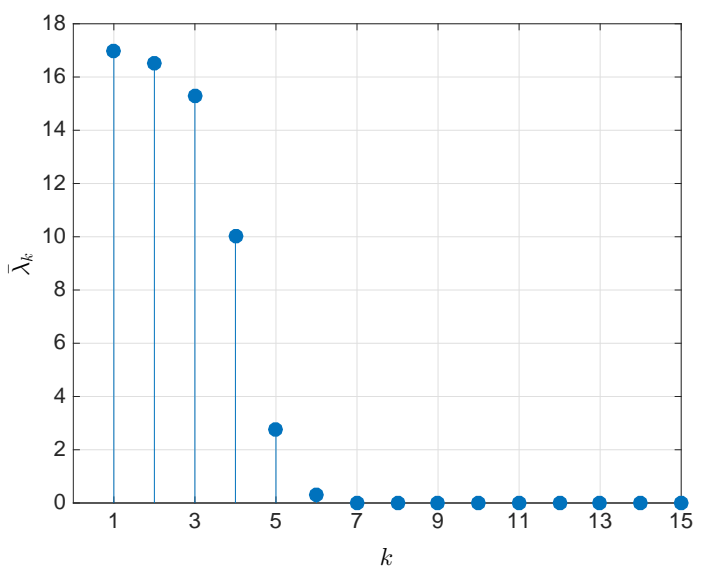

Fig. 3. Eigenvalues of the mismatched channel covariance matrix.

This intuition is also corroborated by the results of Fig. 4, where $P_{f}$ is shown as a function of $K$ for CM, NCM and their RS counterparts in the ETU scenario. Here, we consider an FDD transmission mode with an SNR fixed to $6 \mathrm{~dB}$. As expected, for small values of $K$ the detection capability of all the considered schemes increases with $K$, until a floor is reached for $K \geq 5$. Since these results suggest that a good trade-off between performance and system complexity can be achieved by letting $K=5$, such a value is adopted in all subsequent simulations.

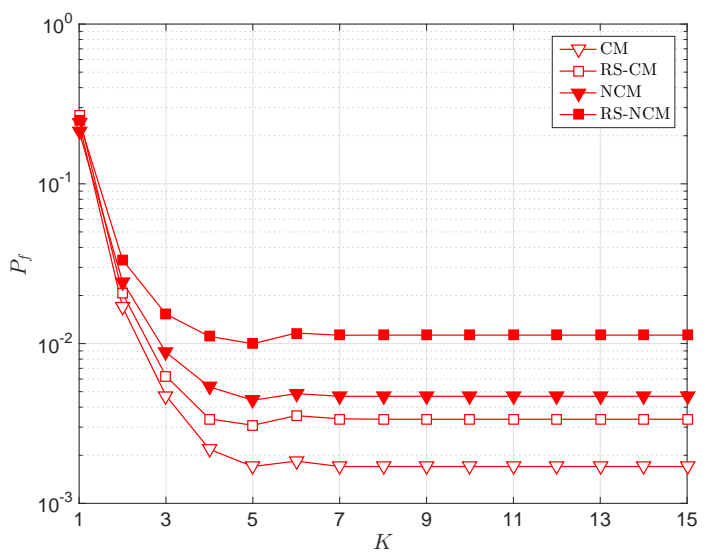

Fig. 4. Probability of detection failure vs. $K$ in the FDD scenario with $\mathrm{SNR}=6 \mathrm{~dB}$.

Fig. 5 illustrates the detection capability of CM, RS-CM, RS-CD and RS-MKBK vs. the SNR. The results are obtained in the FDD scenario using the ETU and EVA channel models. The failure probability of the true ML estimator reported in (29) is also shown for comparison. This scheme can be interpreted as a sort of benchmark to the system performance as it operates with ideal knowledge of the channel covariance matrix. We see that, independently of the considered propagation scenario, CM entails a loss of approximately $1 \mathrm{~dB}$ wth respect to the benchmark as a consequence of the mismatch between the true channel statistics and those employed for the design of the SSS detector. A further loss of $1 \mathrm{~dB}$ (with EVA) or $1.5 \mathrm{~dB}$ (with ETU) is incurred when the complete search over all possible pairs $(\tilde{a}, \tilde{b})$ is replaced by the RS approach. In spite of this, RS-CM largely outperforms both RS-CD and RS-MKBK. The reason is that the latter schemes perform coherent detection without taking into account that channel gains over adjacent subcarriers are highly correlated. On the other hand, such a correlation is inherently exploited by CM and RS-CM through the evaluation of vectors $\overline{\mathbf{v}}_{P}$ and $\overline{\mathbf{v}}_{S}(\tilde{a}, \tilde{b})$, thereby leading to a substantial advantage with respect to competing methods. We also see that, when applied to the ETU channel, the investigated schemes perform marginally better than in the EVA scenario. A possible explanation is that, compared to EVA, the ETU propagation medium is characterized by a greater number of multipath components with significant average power, which results into an improved multipath diversity.

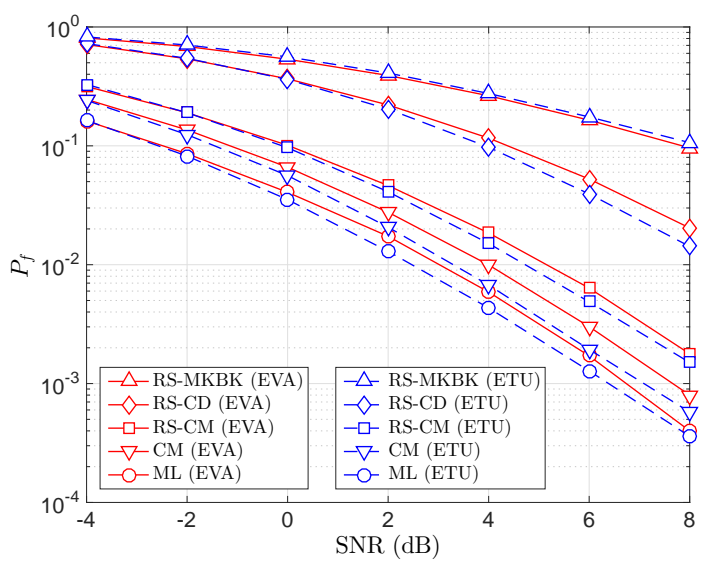

Fig. 5. Probability of detection failure vs. SNR in the FDD scenario using both the P-SSB and S-SSB.

The results shown in Fig. 6 are obtained under the same operating conditions of Fig. 5, except that the LTE network is now operating in the TDD mode. Again, we see that the accuracy of $\mathrm{CM}$ is very close to the true ML estimator, while RS-CM entails a loss of nearly $3 \mathrm{~dB}$. As for RS-CD and RSMKBK, they provide unsatisfactory performance and cannot be used in this scenario. Comparing with Fig. 5, it turns out that all the considered SSS detectors perform worse in the TDD mode than in the FDD mode. Such a behaviour can be explained by recalling that in the type 2 TDD frame structure the PSS is located three OFDM symbols apart from the SSS, while in the type 1 FDD frame structure the synchronization sequences are placed in adjacent symbols. Hence, in the FDD mode the recovered PSS can provide more reliable information about the S-SSB channel response than in the TDD mode, especially in a high mobility scenario characterized by fast channel variations. This is especially true for RS-MKBK and RS-CD which, in spite of the increased multipath diversity offered by the ETU channel, perform better in the EVA sce- 
nario due to the reduced UE speed. In contrast, the proposed algorithms still exhibit marginally better detection capability in the ETU scenario due to the larger diversity offered by this channel and also thanks to their improved resilience against fast channel variations, which are accounted for in these schemes by introducing the decorrelating parameter $\alpha$.

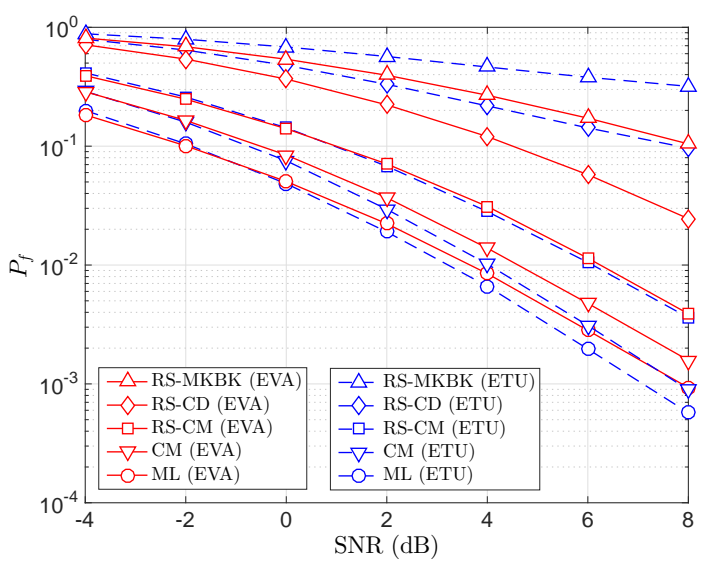

Fig. 6. Probability of detection failure vs. SNR in the TDD scenario using both the P-SSB and S-SSB.

Fig. 7 illustrates the probability of failure of NCM, RSNCM, RS-DD and RS-MEJXD as a function of the SNR. In such a case, the performance is independent of the duplexing mode since only the S-SSB is used to detect the SSS. The trend is similar to that observed in Figs. 5 and 6, with the proposed detectors outperforming all the other considered methods. In particular, the gain of RS-NCM over the RS-DD is approximately $3 \mathrm{~dB}$ in the ETU scenario, and increases to 4 dB with EVA. As for RS-MEJXD, it cannot work in both the investigated channel models. The reason is that this scheme is designed for a flat fading channel and, accordingly, it performs poorly in a severe multipath environment or in the presence of a non-negligible timing error, which results into a linearly increasing phase shift of the S-SSB subcarriers. In general, we see that the considered schemes provide marginally better results in the ETU channel due to the increased multipath diversity with respect to EVA.

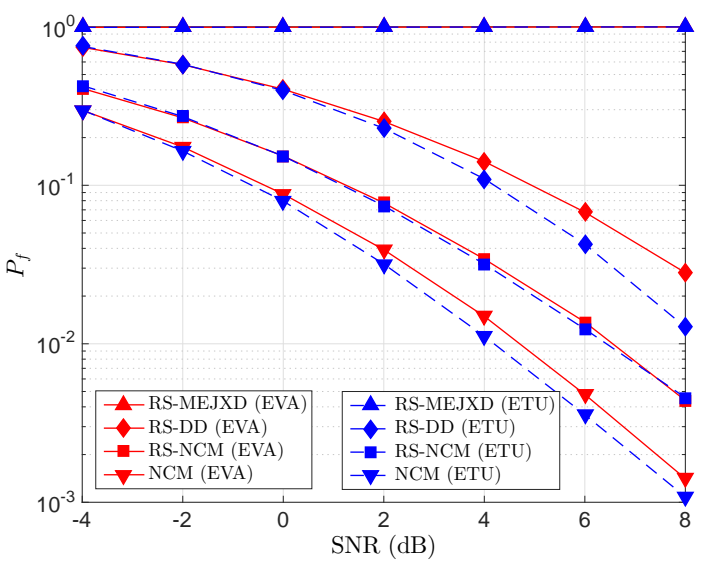

Fig. 7. Probability of detection failure vs. SNR using only the S-SSB.
The sensitivity of NCM, RS-NCM, RS-DD and RS-MEJXD to residual timing errors is assessed in Fig. 8 by measuring $P_{f}$ as a function of $\theta$. For this purpose, an ETU scenario is assumed with the SNR being fixed to $6 \mathrm{~dB}$. These results demonstrate the robustness of all the considered algorithms against timing errors except for RS-MEJXD, which exhibits a remarkable sensitivity to $\theta$. Such a behaviour can be explained by observing that the phase shift impressed by the timing error on the S-SSB subcarriers leads to a loss of coherence of the terms $\left\{Y_{S, \text { even }}(n) s_{\tilde{a}}(n)\right\}$ and $\left\{Y_{S, \text { odd }}(n) s_{\tilde{b}}(n) z_{\hat{a}}(n)\right\}$ employed to evaluate the MEJXD metrics shown in (46) and (48). Other simulation results (not shown for space limitations) indicate that CM, RS-CM, RS-CD and RS-MKBK are particularly resilient against timing errors in both the ETU and EVA scenarios.

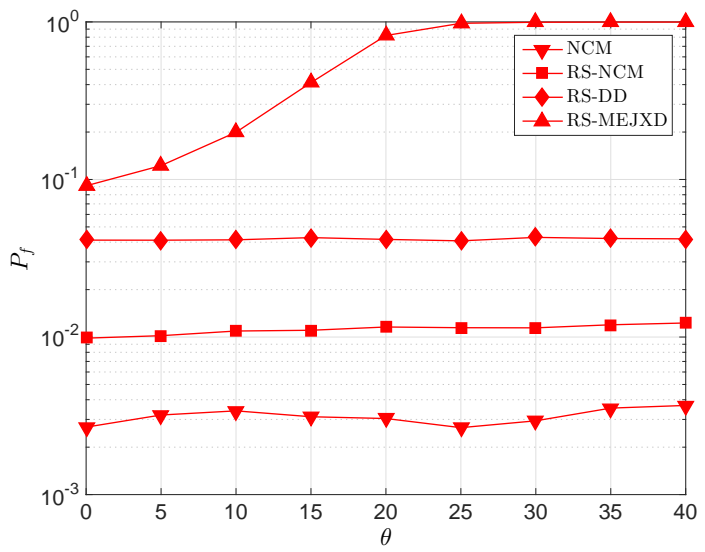

Fig. 8. Probability of detection failure vs. $\theta$ with $\mathrm{SNR}=6 \mathrm{~dB}$ using only the S-SSB.

Fig. 9 shows the probability of failure of RS-CM and RS$\mathrm{CD}$ as a function of the UE speed $v$ for a TDD system. Here, the SNR is $6 \mathrm{~dB}$ and two different values of $\bar{\alpha}$ are used for RS-CM. The first one is computed from (31) assuming a maximum mobile speed $v_{\max }=60 \mathrm{~km} / \mathrm{h}$, while the other value corresponds to a maximum speed $v_{\max }=300$ $\mathrm{km} / \mathrm{h}$. These results indicate that, designing the RS-CM for the worst-case scenario of $v_{\max }=300 \mathrm{~km} / \mathrm{h}$, leads to a probability of failure which remains approximately constant over a wide range of UE speeds, thereby providing the system with increased resilience against fast channel variations. On the other hand, when the reference value $v_{\max }=60 \mathrm{~km} / \mathrm{h}$ is used for the design of $\bar{\alpha}$, an improvement of the system performance is observed at UE speeds lower than $150 \mathrm{~km} / \mathrm{h}$, while a certain degradation occurs at higher speed values. As expected, the detection capability of RS-CD steadily reduces as the UE speed grows large.

\section{Complexity comparison}

We conclude our study by comparing the investigated schemes in terms of their computational complexity. Recalling that the value $K=5$ has been used for the design of the proposed SSS detectors, from the results shown in Table I it turns out that $\mathrm{CM}$ and $\mathrm{NCM}$ require nearly $500 \mathrm{kflops}$ and, accordingly, they are much more complex than the other 


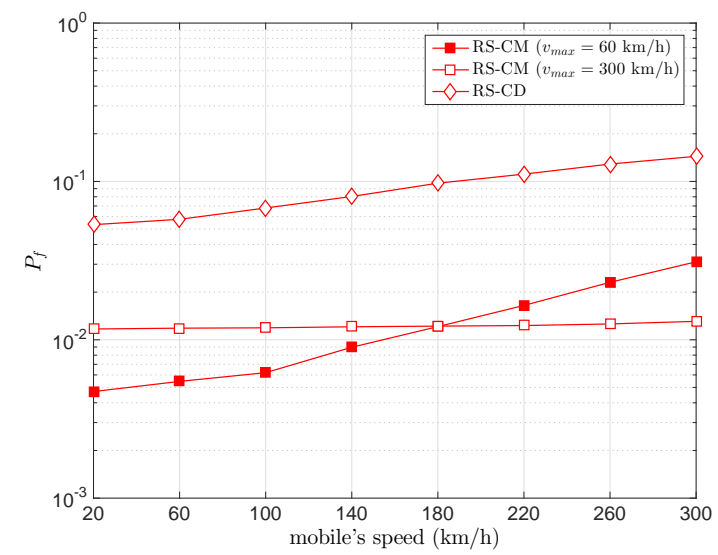

Fig. 9. Probability of detection failure vs. mobile's speed in the TDD scenario using both the P-SSB and S-SSB with $\mathrm{SNR}=6 \mathrm{~dB}$.

considered methods. This represents a strong argument against the complete search approach, which cannot be implemented with affordable complexity. We also see that the improved detection capability of RS-CM and RS-NCM over the other selected alternatives is achieved at the price of a higher processing load. In particular, the number of flops required by these schemes is nearly increased by a factor of 10 with respect to RS-MKBK and by a factor of 5 with respect to RS$\mathrm{CD}$ and RS-DD. The RS-MEJXD turns out to be the simplest method, with only 2.65 kflops required. However, in view of the remarkable gain achieved by RS-CM and RS-NCM over the other methods, their use should be considered despite the penalty incurred in terms of processing requirement.

\section{CONCLUSIONS}

We have presented an ML procedure for SSS detection in the downlink of an LTE system operating in either the FDD or TDD mode. Two different situations have been considered. In the first one, channel state information is extracted from both the P-SSB and S-SSB, while only the S-SSB is exploited in the other one. Since the true ML algorithms require ideal knowledge of the channel statistics, a simplified approach has been considered wherein the system is allowed to operate in a mismatched mode. A RS strategy has also been suggested to further reduce the system complexity. These simplifications lead to the design of two novel ML-oriented schemes (RS-CM and RS-NCM) characterized by a loss of approximately 2 or $3 \mathrm{~dB}$ with respect to the optimal ML solution.

Compared to existing alternatives, the proposed algorithms provide a remarkable SNR gain by properly taking into account the statistical correlation among channel gains over different subcarriers and possible channel variations related to the UE mobility. The price for such an advantage is a certain increase of the processing load. The penalty in terms of required flops is justified by the fact that RS-CM and RS-NCM exhibit good detection capability even in a harsh propagation environment characterized by high-mobility and prolonged delay spreads, where other competing schemes provide poor performance.

\section{REFERENCES}

[1] 3GPP TS 36.211 V10.4.0: Evolved Universal Terrestrial Radio Access (E-UTRA); Physical Channels and Modulation (Release 10), 2011-12.

[2] E. W. Yi-Pin and O. Tony, "Cell search in W-CDMA," IEEE J. Sel. Areas Commun, vol. 18, n. 8, pp. 1470-1482, Aug. 2000.

[3] P. Y. Tsai and H. W. Chang, "A new cell search scheme in 3GPP Long Term Evolution downlink OFDMA systems," in Proc. of 12th Int. Conf. on Wireless Commun. and Signal Proc. (WCSP) 2009, Nanjing, Nov. 2009.

[4] F. Wang, Y. Zhu, and Z. Wang, "A low complexity scheme for S-SCH detection in 3GPP LTE downlink system," in Proc. of IEEE Global Mobile Congress (GMC), 2010.

[5] C.-C. Liao, P.-Y. Tsai and T.-D. Chiueh, "Low-complexity cell search algorithm for interleaved concatenation ML-sequences in 3GPP-LTE systems," IEEE Wireless Commun. Letters, vol. 1, n. 4, pp. 280-283, Aug. 2012.

[6] A. Golnari, G. Sharifan, Y. Amini, and M. Shabany, "A low complexity architecture for the cell search applied to the LTE systems," in Proc. of 19th IEEE Int. Conf. on Electronics, Circuits and Systems (ICECS), pp. 300-303, 2012.

[7] K. Manolakis, D. M. G. Estévez, V. Jungnickel, W. Xu, and C. Drewes, "A closed concept for synchronization and cell search in 3GPP LTE systems," in Proc. of Wireless Commun. and Net. Conf. (WCNC) 2009, Budapest, Apr. 2009.

[8] J.-In Kim, J.-Su Han, H.-J. Roh, and H.-J. Choi, "SSS detection method for initial cell seach in 3 GPP LTE FDD/TDD dual mode receiver," in Proc. of 9th Int. Symp. on Commun. and Inf. Techn. (ISCIT), pp. 199-203, 2009.

[9] J. Myung, J. Kang, Y. Baek, and B. Koo, "Efficient S-SCH detection algorithm for LTE downlink channel," IEEE Trans. on Vehicular Techn., vol. 63, n. 6, pp. 2969-2973, July 2014.

[10] B. Li, G. Sun, and X. Wang, "SSS detection scheme for neighbor cell search in 3GPP LTE systems," in Proc. of Global Telecommun. Conf. (GLOBECOM), 2011.

[11] M. Morelli and M. Moretti, "A robust maximum likelihood scheme for PSS detection and integer frequency offset recovery in LTE systems," IEEE Trans. on Wireless Commun., vol. 15, n. 2, pp. 1353-1363, Feb. 2016.

[12] M. Morelli and M. Moretti, "ML estimation of timing, integer frequency and primary sequence index in LTE systems," in Proc. of IEEE Internat. Conf. on Commun. (ICC), London, June 2015.

[13] J. J. van de Beek, M. Sandell, and P. O. Borjesson, "ML estimation of timing and frequency offset in OFDM systems," IEEE Trans. on Signal Processing, vol. 45, n. 7, pp. 1800-1805, July 1997.

[14] O. Edfors, M. Sandell, J. J. van de Beek, S. K. Wilson, and P. O. Börjesson, "OFDM channel estimation by singular value decomposition," IEEE Trans. on Commun., vol. 46, n. 7, pp.931-939, July 1998.

[15] W. C. Jakes, Microwave Mobile Communications, New-York: Wiley, 1974.

[16] A. Golnari, M. Shabany, A. Nezamalhosseini, and G. Gulak, "Design and implementation of time and frequency synchronization in LTE,' IEEE Trans. on very large scale int. (VLSI) systems, vol. 23, n.12, pp. 2970-2982, Dec. 2015 